\title{
Neighborhood of a Rational Curve with a Node
}

By

\author{
Tetsuo UEDA*
}

\section{§. Introduction and Statement of the Result}

Let $C$ be an irreducible compact analytic set of dimension 1 in a complex manifold of dimension 2. Let $\left(C^{2}\right)$ denote the self-intersection number of $C$. It is well known that $C$ has a strongly pseudoconvex neighborhood if and only if $\left(C^{2}\right)<0$; and that $C$ has a fundamental system of strongly pseudoconcave neighborhoods if $\left(C^{2}\right)>0$. When $\left(C^{2}\right)=0$, this topological condition alone is insufficient to derive analytic conclusions. For a smooth curve $C$ with $\left(C^{2}\right)=$ 0 , we obtained some conditions for the existence of a fundamental system of strongly pseudoconcave or pseudoflat neighborhoods in [8] (see also Neeman [5]).

It will be natural to investigate such complex analytic properties in the case where $C$ has singularities. In the present paper we treat one of the simplest cases of such $C$.

In the sequel, $C$ will always stand for a rational curve with only one node (ordinary double point). To state the result, we note first that the Picard variety of $C$, i.e., the set of all topologically trivial line bundles over $C$, is isomorphic to $H^{1}\left(C, C^{*}\right) \cong C^{*}$ as multiplicative group (see Lemma 1 ).

Suppose that $C$ is imbedded in a complex manifold $S$ of dimension 2 and $\left(C^{2}\right)=0$. Let $[C]$ denote the line bundle over $S$ associated to the divisor $C$. The normal bundle $N$ of $C$ is defined to be the restriction $[C] \mid C$ of $[C]$ to $C$. By the assumption, $N$ is a topologically trivial line bundle over $C$. Let $\alpha \in C^{*}$ be the number corresponding to $N$ by the above isomorphism. Let $d(p)$ denote the distance of $p \in S$ from the curve $C$ with respect to some Riemannian metric

Communicated by K. Saito, October 15, 1990.

1991 Mathematics Subject Classification: 32F15.

* Institute of Matheınatics, Yoshida College, Kyoto University, Kyoto, 606 Japan. 
on $S$. The main result of the present paper consists of the following theorems.

Theorem 1. Suppose $|\alpha| \neq 1$. Then there exist a neighborhood $V$ of $\mathbb{C}$ and a strongly plurisubharmonic function $\Phi$ on $V-C$ such that $\Phi(p) \rightarrow+\infty$ as $p \rightarrow C$. Moreover, for any real number $\lambda>1$, we can construct $\Phi$ so that $\Phi(p) /\left(\log \frac{1}{d(p)}\right)^{2 \lambda}$ is bounded.

Theorem 2. Suppose $|\alpha| \neq 1$. Let $V^{\prime}$ be a neighborhood of $C$ and $\Psi(p)$ a plurisubharmonic function on $V^{\prime}-C$ such that $\Psi(p) /\left(\log \frac{1}{d(p)}\right)^{2 \lambda}$ is bounded for some real number $\lambda<1$. Then there is a neighborhood $V_{0}$ of $\mathbb{C}$ such that $\Psi \mid\left(V_{0}-C\right)$ is constant.

These results correspond to Theorems 1 and 2 in [8] (for smooth curves of finite type). We note that, in the present case, the neighborhood of $C$ admits plurisubharmonic functions with slower growth than in the case of [8].

Theorems 1 and 2 are proved in $\S 3$, after some preliminaries in $\S 1$ and $\S 2$. We consider, in $\S 4$, such curves in compact complex surfaces. Examples of surfaces of class $\mathrm{VII}_{0}$ and rational surfaces are given.

\section{Acknowledgement}

The author would like to thank Professors T. Ohsawa and H. Yamaguchi for valuable suggestions.

\section{§1. Topologically Trivial Line Bundles over $\mathbb{C}$}

Let $C$ be a rational curve with only one node $p_{0}$. Let $\mathbb{P}$ denote a smooth rational curve (Riemann sphere) with inhomogeneous coordinate $\zeta$ and let $\iota: \mathbb{P} \rightarrow C$ be a desingularization map of $C$ such that $\iota(0)=\imath(\infty)=p_{0}$. We fix $\varepsilon$ with $0<\varepsilon<1$ and define open subsets $U_{i}(i=0,1)$ of $C$ by

$$
U_{0}=U_{0}^{+} \cup U_{0}^{-}
$$

where

$$
U_{0}^{+}=\iota(\{|\zeta|<\varepsilon\}), \quad U_{0}^{-}=\iota(\{1 / \varepsilon<|\zeta| \leqq \infty\})
$$

and

$$
U_{1}=\iota(\{0<|\zeta|<\infty\}) .
$$

Then $\left\{U_{0}, U_{1}\right\}$ is an open covering of $C$. The intersection $U_{0} \cap U_{1}$ consists of two connected components 


$$
U^{+}=\iota(\{0<|\zeta|<\varepsilon\}), \quad U^{-}=\iota(\{(1 / \varepsilon<|\zeta|<\infty\}) .
$$

Every holomorphic line bundle over $C$ is represented by a multiplicative 1-cocycle with respect to the covering $\left\{U_{i}\right\}$. We denote this 1 -cocycle by a pair $\left(f^{+}, f^{-}\right)$of non-vanishing holomorphic functions $f^{ \pm}$on $U^{ \pm}$.

Lemma 1. Every topologically trivial line bundle $E$ over $C$ is represented by a pair $(1, \alpha)$ where $\alpha$ is a complex number $\neq 0$ uniquely determined by $E$. In other words, the Picard variety of $C$ is isomorphic to $H^{1}\left(C, C^{*}\right) \cong C^{*}$.

Proof. Suppose that $E$ is represented by a pair $\left(f^{+}, f^{-}\right)$. We regard $f^{ \pm}$ as functions of the variable $\zeta$ with $0<|\zeta|<\varepsilon$ and $1 / \varepsilon<|\zeta|<\infty$, respectively. Let

$$
m^{+}=\frac{1}{2 \pi i} \int_{|\zeta|=\varepsilon / 2} \frac{d f^{+}}{f^{+}}, \quad m^{-}=\frac{1}{2 \pi i} \int_{|\zeta|=2 / \varepsilon} \frac{d f^{-}}{f^{-}} .
$$

Then we can write

$$
f^{ \pm}=\zeta^{m \pm} \exp g^{ \pm}
$$

where $g^{+}, g^{-}$are holomorphic functions on $U^{+}, U^{-}$. By Laurent expansion, they are decomposed as follows:

$$
\begin{aligned}
& g^{+}=a_{0}^{+}+g_{+}^{+}+g_{-}^{+} \\
& g^{-}=a_{0}^{-}+g_{+}^{-}+g_{-}^{-}
\end{aligned}
$$

with

$$
\begin{array}{ll}
g_{+}^{+}(\zeta)=\sum_{k>0} a_{k}^{+} \zeta^{k}, & g_{-}^{+}(\zeta)=\sum_{k<0} a_{k}^{+} \zeta^{k} \\
g_{+}^{-}(\zeta)=\sum_{k>0} a_{k}^{-} \zeta^{k}, & g_{-}^{-}(\zeta)=\sum_{k<0} a_{k}^{-} \zeta^{k} .
\end{array}
$$

The power series $g_{+}^{+}, g_{-}^{+}, g_{+}^{-}, g_{-}^{-}$define holomorphic functions for $|\zeta|<\varepsilon$, $0<|\zeta| \leqq \infty,|\zeta|<\infty, 1 / \varepsilon<|\zeta| \leqq \infty$, respectively.

We define non-vanishing holomorphic functions $f_{i}$ on $U_{i}, i=0,1$, by

$$
\begin{aligned}
& f_{0}= \begin{cases}\exp \left(g_{+}^{+}-g_{+}^{-}\right) & \text {for }|\zeta|<\varepsilon, \\
\exp \left(-g_{-}^{+}+g_{-}^{-}\right) & \text {for } 1 / \varepsilon<|\zeta| \leqq \infty,\end{cases} \\
& f_{1}=\zeta^{-m^{+}} \exp \left(-a_{0}^{+}-g_{-}^{+}-g_{+}^{-}\right) \text {for } 0<|\zeta|<\infty .
\end{aligned}
$$

Then we have

$$
\left\{\begin{array}{lll}
f^{+} f_{1} / f_{0}=1 & \text { on } & U^{+} \cong\{0<|\zeta|<\varepsilon\}, \\
f^{-} f_{1} / f_{0}=\alpha \zeta^{m} & \text { on } & U^{-} \cong\{1 / \varepsilon<|\zeta|<\infty\},
\end{array}\right.
$$


where $m=m^{-}-m^{+}, \alpha=\exp \left(a_{0}^{-}-a_{0}^{+}\right)$. This shows that the line bundle $E$ is represented by the pair $\left(1, \alpha \zeta^{m}\right)$.

Now, since $E$ is topologically trivial, the pull-back $\iota^{*}(E)$ of $E$ by $\iota: \mathbb{P} \rightarrow C$ is also topologically trivial. $\iota^{*}(E)$ is represented by the 1 -cocycle $\zeta^{m} \alpha$ with respect to the covering $\{|\zeta|<\infty\},\{1 / \varepsilon<|\zeta| \leqq \infty\}$ of $\mathbb{P}$. Hence we have $m=0$.

q.e.d.

Remark 1. This correspondence depends on the choice of the coordinate

$\zeta:$ If we replace $\zeta$ by $1 / \zeta$, then $\alpha$ is replaced by $1 / \alpha$ 。

\section{§2. Coordinate Systems on the Neighborhood of $\mathbb{C}$}

Now suppose that $C$ is imbedded in a complex manifold of dimension 2 and that $\left(C^{2}\right)=0$.

We choose neighborhoods $V_{0}, V_{1}$ of $U_{0}, U_{1}$ such that $C \cap V_{8}=U_{i}$, and that $V_{0} \cap V_{1}$ consists of two connected components $V^{+}, V^{-}$with $C \cap V^{+}=U^{+}$, $C \cap V^{-}=U^{-}$. Further we suppose that $V_{i}$ are Stein open sets. This is possible by a theorem of $\mathrm{Siu}$ [7]. In the following consideration, $V_{0}, V_{1}$ will be replaced by smaller ones whenever it is necessary.

Lemma 2. Suppose that $\alpha$ is not a root of unity. Then, for any integer $\nu \geqq 1$, there exist holomorphic functions $w_{i}$ on $V_{i}(i=0,1)$ such that $w_{i}=0$ are defining equations of $C$ in $V_{i}$ and satisfying the conditions:

(i) $w_{1}-w_{0}$ vanishes to order $\nu+1$ on $U^{+}$,

(ii) $w_{1}-\alpha w_{0}$ vanishes to order $\nu+1$ on $U^{-}$.

Proof. First we prove the lemma for $\nu=1$. Let $w_{i}$, be any holomorphic functions on $V_{i}$ such that $w_{i}=0$ are defining equations of $C$ in $V_{i}(i=0,1)$. We want to modify $w_{i}$ and obtain $\tilde{w}_{i}$ satisfying the conditions (i) and (ii). We write $w_{1}=F^{+} w_{0}$ on $V^{+}$and $w_{1}=F^{-} w_{0}$ on $V^{-}$with non-vanishing functions $F^{ \pm}$ on $V^{ \pm}$. Let $f^{ \pm}=F^{ \pm} \mid U^{ \pm}$. The pair $\left(f^{+}, f^{-}\right)$represents the complex normal bundle $N$ of $C$. By Lemma 1, there exist non-vanishing holomorphic functions $f_{i}$ on $U_{i}(i=0,1)$ satisfying the equations $(1)$, with $m=0$. We choose nonvanishing holomorphic functions $F_{i}$ on $V_{i}$ such that $F_{i} \mid U_{i}=f_{i}$ and put $\tilde{w}_{i}=$ $F_{i} w_{i}(i=0,1)$. Then $\tilde{w}_{0}, \tilde{w}_{1}$ have the required properties for $\nu=1$ as easily verified.

For $\nu \geqq 2$, we proceed by induction. Assume there are holomorphic functions $w_{i}$ as in the lemma for some integer $\nu(\geqq 1)$. Then we can write 


$$
\begin{array}{ll}
w_{1}=w_{0}+H^{+} w_{0}^{\nu+1} & \text { on } \quad V^{+}, \\
w_{1}=\alpha\left(w_{0}+H^{-} w_{0}^{\nu+1}\right) & \text { on } \quad V^{-}
\end{array}
$$

with holomorphic functions $H^{ \pm}$on $V^{ \pm}$. Let $h^{ \pm}=H^{ \pm} \mid U^{ \pm}$. We want to obtain holomorphic functions $h_{i}$ on $U_{i}(i=0,1)$ satisfying the equations

$$
\left\{\begin{array}{lll}
h_{0}-h_{1}=h^{+} & \text {on } U^{+}, \\
h_{0}-\alpha^{2} h_{1}=h^{-} & \text {on } & U^{-} .
\end{array}\right.
$$

To solve (2) we decompose $h^{ \pm}$as follows:

$$
\begin{aligned}
& h^{+}=b_{0}^{+}+h_{+}^{+}+h_{-}^{+}, \\
& h^{-}=b_{0}^{-}+h_{+}^{-}+h_{-}^{-},
\end{aligned}
$$

where

$$
\begin{array}{ll}
h_{+}^{+}(\zeta)=\sum_{k>0} b_{k}^{+} \zeta^{k}, & h_{-}^{+}(\zeta)=\sum_{k<0} b_{k}^{+} \zeta^{k} \\
h_{+}^{-}(\zeta)=\sum_{k>0} b_{k}^{-} \zeta^{k}, & h_{-}^{-}(\zeta)=\sum_{k<0} b_{k}^{-} \zeta^{k} .
\end{array}
$$

The power series $h_{+}^{+}, h_{-}^{+}, h_{+}^{-}, h_{-}^{-}$define holomorphic functions for $|\zeta|<\varepsilon$, $0<|\zeta| \leqq \infty,|\zeta|<\infty, 1 / \varepsilon<|\zeta| \leqq \infty$, respectively.

We define

$$
\begin{aligned}
& h_{0}=\left\{\begin{array}{lll}
c_{0}+h_{+}^{+}-\alpha^{-\nu} h_{+}^{-} & \text {on } \quad U_{0}^{+} \cong\{|\zeta|<\varepsilon\}, \\
c_{0}-\alpha^{\nu} h_{-}^{+}+h_{-}^{-} & \text {on } \quad U_{0}^{-} \cong\{1 / \varepsilon<|\zeta| \leqq \infty\}
\end{array}\right. \\
& h_{1}=c_{1}-h_{-}^{+}-\alpha^{-\nu} h_{+}^{-} \quad \text { on } \quad U_{1} \cong\{0<|\zeta| \leqq \infty\} \text {, }
\end{aligned}
$$

where $c_{0}=\frac{\alpha^{\nu} b_{0}^{+}-b_{0}^{-}}{\alpha^{\nu}-1}, \quad c_{1}=\frac{b_{0}^{+}-b_{0}^{-}}{\alpha^{\nu}-1}$.

Then $h_{0}, h_{1}$ satisfy the equations (2).

Now let $H_{i}$ be holomorphic functions on $V_{i}$ such that $H_{i} \mid U_{i}=h_{i}(i=0,1)$. We define

$$
\begin{array}{lll}
\tilde{w}_{0}=w_{0}+H_{0} w_{0}^{\nu+1} & \text { on } & V_{0} ; \\
\tilde{w}_{1}=w_{1}+H_{1} w_{1}^{\nu+1} & \text { on } & V_{1} .
\end{array}
$$

Then $\tilde{w}_{0}, \tilde{w}_{1}$ satisfy the conditions (i), (ii) with $\nu+1$ in place of $\nu$. In fact, on $V^{+}$, we have

$$
\begin{aligned}
\tilde{w}_{1}-\tilde{w}_{0} & =H^{+} w_{0}^{\nu+1}+H_{1} w_{1}^{\nu+1}-H_{0} w_{0}^{\nu+1} \\
& =\left(H^{+}+H_{1}-H_{0}\right) w_{0}^{\nu+1}+H_{1}\left\{\left(w_{0}+H^{+} w_{0}^{\nu+1}\right)^{\nu+1}-w_{0}^{\nu+1}\right\} .
\end{aligned}
$$

Since $H^{+}+H_{1}-H_{0}$ vanishes on $U^{+}$by the first equality of (2), $\tilde{w}_{1}-\tilde{w}_{0}$ vanishes to order $\nu+2$ on $U^{+}$. On $V^{-}$, we have 


$$
\begin{aligned}
\tilde{w}_{1}-\alpha \tilde{w}_{0} & =\alpha H^{-} w_{0}^{\nu+1}+H_{1} w_{1}^{\nu+1}-\alpha H_{0} w_{0}^{\nu+1} \\
& =\alpha\left(H^{-}+\alpha^{\nu} H_{1}-H_{0}\right) w_{0}^{\nu+1}+\alpha^{\nu+1} H_{1}\left\{\left(w_{0}+H^{-} w_{0}^{\nu+1}\right)^{\nu+1}-w_{0}^{\nu+1}\right\}
\end{aligned}
$$

Since $H^{-}+\alpha^{\nu} H_{1}-H_{0}$ vanishes on $U^{-}$by the second equality of (2), $\tilde{w}_{1}-\alpha \tilde{w}_{0}$ vanishes to order $\nu+2$ on $U^{-}$.

q.e.d.

Remark 2. We can extend the normal bundle $N$ to a complex line bundle $F$ over $V=V_{0} \cup V_{1}$ by extending the 1 -cocycle $(1, \alpha)$ to $V^{+}, V^{-}$as constant. Then Lemma 2 implies that the bundles [C] and $F$ coincide formally along the curve $\mathbb{C}$.

Now let $\zeta: U_{1} \rightarrow \mathbb{P}$ denote the inverse of the biholomorphic map $\iota \mid(\mathbb{P}-\{0, \infty\}): \mathbb{P}-\{0, \infty\} \rightarrow U_{1}$.

Lemma2 3. There exist holomorphic functions $z_{i}$ on $V_{i}(i=0,1)$ satisfying the following conditions:

(i) $z_{1} \mid U_{1}=\zeta$;

(ii) $z_{1}=B^{+} z_{0}$ on $V^{+}$, where $B^{+}$is a holomorphic function on $V^{+}$such that $B^{+} \mid U^{+}=1$;

(iii) $z_{1}=B^{-} z_{0} / w_{0}$ on $V^{-}$, where $B^{-}$is a holomorphic function on $V^{-}$such that $B^{-} \mid U^{-}$is a constant $\neq 0$.

Proof. We choose and fix as $z_{1}$ any holomorphic function on $V_{1}$ satisfying the condition (i). Let $z_{0}$ be a holomorphic function on $V_{0}$ which takes zero of order 1 on $U_{0}^{-}$and such that $z_{0} \mid U_{0}^{+}=\zeta$. Then $z_{0}, z_{1}$ satisfy the conditions (i), (ii). We wish to modify $z_{0}$ and obtain $\tilde{z}_{0}$ so that the condition (iii) is also satisfied by $\tilde{z}_{0}, z_{1}$.

Let $Q=z_{0} / w_{0}$. Then $Q$ is a meromorphic function on $V_{0}$ which has simple poles on $U_{0}^{+}$and has no other poles nor zeros. Let $q=Q \mid U_{0}^{-}$and $r=q / \zeta$. Then $r$ is extended to a non-vanishing holomorphic function on $U_{0}^{+}$. Let $\beta=$ $r\left(p_{0}\right)$ denote the value of the function $r$ at the node $p_{0}$. Choose a non-vanishing holomorphic function $S$ on $V_{0}$ such that $S \mid U_{0}^{+}=1$ and $S \mid U_{0}^{-}=r / \beta$.

We put $\tilde{z}_{0}=z_{0} / S$. Then $\tilde{z}_{0}, z_{1}$ satisfy the conditions of the lemma. In fact, on $V^{+}$, we have $z_{1} / \tilde{z}_{0}=S z_{1} / z_{0}$, whose restriction to $U^{+}$is identically equal to 1 . On $V^{-}$, we have $z_{1} w_{0} / \tilde{z}_{0}=S z_{1} w_{0} / z_{0}=S z_{1} / Q$, whose restriction to $U_{0}^{-}$is $(r \zeta) /(\beta q)=$ $1 / \beta$.

q.e.d.

\section{§3. Proofs of the Theorems}

Let $w_{i}(i=0,1)$ be as in Lemma 2 , with $\nu=4$. Then we have $w_{1}=A^{+} w_{0}$ 
on $V^{+}$and $w_{1}=\alpha A^{-} w_{0}$ on $V^{-}$, where $A^{ \pm}$are holomorphic functions on $V^{ \pm}$ such that $A^{ \pm}-1$ vanishes to order 4 on $C$. Let $z_{i}(i=0,1)$ be as in Lemma 3 . Here we assume that $B^{-} \mid U^{-}=1$ in the condition (iii). This is possible since we may multiply $w_{i}$ by a constant.

We define real-valued functions $\varphi_{i}$ on $V_{i}-U_{i}$ by

$$
\varphi_{i}=\left(\log \left|w_{i}\right|\right)^{2}-\log |\alpha| \log \left|w_{i}\right|+2 \log |\alpha| \log \left|z_{i}\right|, \quad i=0,1
$$

The difference $\varphi_{1}-\varphi_{0}$ is extended to a function of $C^{2}$ class on $V_{0} \cap V_{1}$ which vanishes on $C \cap V_{0} \cap V_{1}$.

In fact, we have $w_{1}=A^{+} w_{0}, z_{1}=B^{+} z_{0}$ on $V^{+}$and

$$
\varphi_{1}-\varphi_{0}=2 \log \left|A^{+}\right| \log \left|w_{0}\right|+\left(\log \left|A^{+}\right|\right)^{2}-\log |\alpha| \log \left|A^{+}\right|+2 \log |\alpha| \log \left|B^{+}\right| .
$$

The term $2 \log \left|A^{+}\right| \log \left|w_{0}\right|$ is extended to a function of $C^{2}$ class by setting to be 0 on $C$, since $\log \left|A^{+}\right|$vanishes to order 4 on $U^{+}$; and the other terms are real-analytic. This shows the assertion for $V^{+}$. Now we write $\hat{w}_{0}=\alpha w_{0}$, $\hat{z}_{0}=z_{0} / w_{0}$. Then, by straightforward calculation we have

$$
\varphi_{0}=\left(\log \left|\hat{w}_{0}\right|\right)^{2}-\log |\alpha| \log \left|\hat{w}_{0}\right|+2 \log |\alpha| \log \left|\hat{z}_{0}\right| .
$$

Noting that $w_{1}=A^{-} \hat{w}_{0}, z_{1}=B^{-} \hat{z}_{0}$ on $V^{-}$, we can verify the assertion similarly for $V^{-}$.

Now let $V_{0}^{\prime}$ be a neighborhood of the node $p_{0}$ which is relatively compact in $V_{0}$, and let $\rho$ be a real-valued function of $C^{\infty}$ class on $V_{0} \cup V_{1}$ such that $0 \leqq \rho(p) \leqq 1, \operatorname{supp}(\rho) \subset V_{0}$, and $\rho \mid V_{0}^{\prime}=1$. We define a function $\varphi$ of $C^{\infty}$ class on $V-C$ by

$$
\varphi=\rho \varphi_{0}+(1-\rho) \varphi_{1}
$$

It is clear that $\varphi(p)$ tends to $+\infty$ as $p$ tends to the curve $C$. We will prove the following assertions:

1. If $\lambda>1$, then there is a sufficiently small neighborhood $V$ of $C$ such that $\varphi^{\lambda}$ is strongly plurisubharmonic on $V-C$.

2. If $0<\lambda<1$, then there is a sufficiently small neighborhood $V$ of $C$ such that the complex Hessian of $\varphi^{\lambda}$ has one positive and one negative eigenvalues on $V-C$.

The assertion 1 implies Theorem 1 . Theorem 2 is derived from the assertion 2 in the same manner as in $[8, \S 3.4]$.

To prove the assertions, we denote 


$$
H(\psi)=\left[\begin{array}{ll}
\psi_{w \bar{w}} & \psi_{w \bar{z}} \\
\psi_{z \bar{w}} & \psi_{z \bar{z}}
\end{array}\right], \quad G(\psi)=\left[\begin{array}{ll}
\left|\psi_{w}\right|^{2} & \psi_{w} \psi_{\bar{z}} \\
\psi_{z} \psi_{\bar{w}} & \left|\psi_{z}\right|^{2}
\end{array}\right]
$$

for a real-valued function $\psi(w, z)$. Then the complex Hessian $H\left(\varphi^{\lambda}\right)$ of $\varphi^{\lambda}$ is

$$
H\left(\varphi^{\lambda}\right)=\lambda \varphi^{\lambda-2}(\varphi H(\varphi)+(\lambda-1) G(\varphi)) .
$$

First we look at the neighborhood of $U_{1}=C-\left\{p_{0}\right\}$. We choose a sufficiently small neighborhood $V_{1}$ of $U_{1}$ so that $\left(w_{1}, z_{1}\right)$ is a coordinate system on $V_{1}$. On $V_{1}, \varphi$ has the form

$$
\varphi=\left(\log \left|w_{1}\right|\right)^{2}-\log |\alpha| \log \left|w_{1}\right|+\eta,
$$

where

$$
\eta=2 \log |\alpha| \log \left|z_{1}\right|+\rho\left(\varphi_{0}-\varphi_{1}\right) .
$$

The function $\eta$ is of $C^{2}$ class on $V_{1}$ and $\eta_{z_{1}}=(\log |\alpha|) / z_{1} \neq 0, \eta_{z_{1} z_{1}}=0$ on $C$. From

$$
\begin{aligned}
& H(\varphi)=\left[\begin{array}{cc}
\frac{1}{2\left|w_{1}\right|^{2}}+\eta_{w_{1} \bar{w}_{1}} & \eta_{w_{1} \bar{z}_{1}} \\
\eta_{z_{1} \bar{w}_{1}} & \eta_{z_{1} \bar{z}_{1}}
\end{array}\right], \\
& G(\varphi)=\left(\begin{array}{cc}
\left(\frac{\log \left|w_{1}\right|}{\left|w_{1}\right|}\right)^{2}(1+o(1)) & \frac{\log \left|w_{1}\right|}{w_{1}}(1+o(1)) \eta_{\bar{z}_{1}} \\
\eta_{z_{1}} \frac{\log \left|w_{1}\right|}{\bar{w}_{1}}(1+o(1)) & \left|\eta_{z_{1}}\right|^{2}
\end{array}\right)
\end{aligned}
$$

and $\varphi=\left(\log \left|w_{1}\right|\right)^{2}(1+o(1))$, we obtain

$$
\begin{aligned}
\frac{1}{\lambda \varphi^{\lambda-2}} H\left(\varphi^{\lambda}\right) & =\varphi H(\varphi)+(\lambda-1) G(\varphi) \\
= & \left(\begin{array}{lc}
\left(\lambda-\frac{1}{2}\right)\left(\frac{\log \left|w_{1}\right|}{\left|w_{1}\right|}\right)^{2}(1+o(1)) & (\lambda-1) \frac{\log \left|w_{1}\right|}{w_{1}} \eta_{\bar{z}_{1}}(1+o(1)) \\
(\lambda-1) \eta_{z_{1}} \frac{\log \left|w_{1}\right|}{\bar{w}_{1}}(1+o(1)) & (\lambda-1)\left|\eta_{z_{1}}\right|^{2}(1+o(1))
\end{array}\right) .
\end{aligned}
$$

Here $o(1)$ denotes the terms which tend to 0 as $p \rightarrow C$. The determinant of this matrix is of the form $\frac{(\lambda-1)}{2}\left(\frac{\log \left|w_{1}\right|}{\left|w_{1}\right|}\right)^{2}\left|\eta_{z_{1}}\right|^{2}(1+o(1))$, and hence positive or negative according as $\lambda>1$ or $\lambda<1$ everywhere on $V_{1}-C$, if $V_{1}$ is sufficiently small.

Now we look at the vicinity of the node $p_{0}$. If $V_{0}^{\prime \prime} \subset V_{0}^{\prime}$ is a sufficiently small neighborhood of $p_{0}$, we can regard $\left(w_{0} / z_{0}, z_{0}\right)$ as a coordinate system on 
$V_{0}^{\prime \prime}$. Hence we can regard $\left(w_{0}, z_{0}\right)$ as a coordinate system on $V_{0}^{\prime \prime}-C$. Noting that $\varphi=\varphi_{0}$ on $V_{0}^{\prime}$, we obtain from (3)

$$
\begin{aligned}
\frac{1}{\lambda \varphi^{\lambda-2}} H\left(\varphi^{\lambda}\right) & =\varphi H(\varphi)+(\lambda-1) G(\varphi) \\
& =\left[\begin{array}{cc}
\frac{\varphi}{2\left|w_{0}\right|^{2}}+(\lambda-1)\left|\varphi_{w_{0}}\right|^{2} & (\lambda-1) \varphi_{w_{0}} \varphi_{\bar{z}_{0}} \\
(\lambda-1) \varphi_{z_{0}} \varphi_{\bar{w}_{0}} & (\lambda-1)\left|\varphi_{z_{0}}\right|^{2}
\end{array}\right],
\end{aligned}
$$

whose determinant $\frac{\varphi}{2\left|w_{0}\right|^{2}}(\lambda-1)\left|\varphi_{z_{0}}\right|^{2}$ is positive or negative according as $\lambda>1$ or $\lambda<1$.

Thus the assertions are shown, and Theorems 1 and 2 are proved.

\section{$\S 4$. Curves on Compact Surfaces}

A complex manifold $X$ is said to be 1-convex (or strongly pseudoconvex), if there is an exhaustion function $\Phi: X \rightarrow \boldsymbol{R}$ which is strongly plurisubharmonic except on a compact set in $X$. If $X$ is non-compact and 1-convex, then there are a compact analytic set $A$, a Stein space $\hat{X}$ and a proper holomorphic mapping $\pi: X \rightarrow \hat{X}$ such that $\pi(A)$ is a discrete set and $\pi \mid X-A$ is a biholomorphic mapping. By Narasimhan [4] we have $H^{2 n-1}(\hat{X}, Z Z)=0$ if $n \geqq 2$. Since $A$ is at most real $(2 n-2)$-dimensional, we obtain $H^{2 n-1}(X, Z)=0$.

Lemma 4. Let $S$ be a compact complex manifold of dimension $n \geqq 2$ and $C$ an analytic set in $S$. If the complement $S-C$ is 1-convex, then the homomorphism $H_{1}(C, \mathbb{Z}) \rightarrow H_{1}(S, \mathbb{Z})$ is surjective.

Proof. We consider the exact sequence

$$
H_{1}(C, Z) \rightarrow H_{1}(S, Z Z) \rightarrow H_{1}(S, C ; Z) .
$$

Since $S-C$ is 1 -convex we have $H^{2 n-1}(S-C, Z)=0$. Hence $H_{1}(S, C ; Z \mathbb{Z})=0$ by duality. This implies the assertion.

Now suppose that $S$ is a compact complex surface and $C$ a rational curve with a node on $S$ satisfying the condition of Theorem 1 . Then $S-C$ is 1 -convex and hence, by Lemma 4 , the first Betti number $b_{1}(S)$ is either 0 or 1 . We will give examples of both cases:

Example 1. Surfaces of class $\mathrm{VII}_{0}$ (minimal compact complex surfaces with $b_{1}(S)=1$ ) containing divisors $D \neq 0$ with $\left(D^{2}\right)=0$ were determined by Enoki 
[1]. By his result, if $S$ is of class $\mathrm{VII}_{0}$ and contains a rational curve $C$ with a node and with $\left(C^{2}\right)=0$, then $S=S_{1, \alpha, t}(0<|\alpha|<1, t \in C)$. It is easy to show that the number corresponding to the normal bundle of $C$, by Lemma 1 , is $\alpha$ (or $1 / \alpha$, see Remark 1 ). Hence, by Theorem 1 , the complement $S-\mathbb{C}$ is 1-convex.

The complement $S-C$ is described as follows [1]. Let $g$ be the holomorphic automorphism of $\mathbb{C} \times \mathbb{C}^{*}$ defined by

$$
g:(z, w) \rightarrow(w z+t, \alpha w) .
$$

Then $S-C$ is biholomorphic to the quotient surface $\left(\mathbb{C} \times \mathbb{C}^{*}\right) /\langle g\rangle$ of $\mathbb{C} \times \mathbb{C}^{*}$ by $g$. We note that, in the case $t=0$, a plurisubharmonic function can be constructed explicity: Since

$$
(\log |w|)^{2}-\log |\alpha| \log |w|+2 \log |\alpha| \log |z|, \quad(z, w) \in \mathbb{C} \times \mathbb{C}^{*},
$$

is invariant under $g$, this defines a function $\varphi$ on $S-C$. The function $\varphi$ is plurisubharmonic and increases with the same order as $\left(\log \frac{1}{d(p)}\right)^{2}$, when $p \rightarrow C$.

Example 2. Let $C_{0}$ be a cubic curve with a node $p_{0}$ in the projective plane $\mathbb{P}^{2}$. Let $\iota_{0}: \mathbb{P} \rightarrow C_{0}$ be a desingularization map of $C_{0}$. We choose an inhomogeneous coordinate $\zeta$ on $\mathbb{P}$ so that $\iota_{0}(0)=\iota_{0}(\infty)=p_{0}$ and that $p_{1}=\iota_{0}(1)$ is one of the three inflexion points of $C_{0}$. Then the normal bundle of $C_{0}$ is $\left[C_{0}\right] \mid C_{0}=9\left[p_{1}\right]$. We choose nine distinct points $\zeta_{1}, \cdots, \zeta_{9} \in \mathbb{C}^{*}=\mathbb{P}-\{0, \infty\}$ and let $q_{j}=\ell_{0}\left(\zeta_{j}\right)$. Blow up $\mathbb{P}^{2}$ at the points $q_{j}$ so that we have a compact surface $S$ with $\pi: S \rightarrow \mathbb{P}^{2}$. Denote by $C$ the proper transform of $C_{0}$. Then the normal bundle $N$ of $C$ is $[C] \mid C=9\left[\hat{p}_{1}\right]-\sum_{j=1}^{9}\left[\hat{q}_{j}\right]$, where $\hat{p}_{1}=\pi^{-1}\left(p_{1}\right) \cap C$ and $\hat{q}_{j}=\pi^{-1}\left(q_{j}\right) \cap C$. Since we have $\alpha(N)=\zeta_{1} \cdots \zeta_{9}$, the complement $S-C$ is 1-convex if $\zeta_{j}$ are so chosen that $\left|\zeta_{1} \cdot \bullet \zeta_{9}\right| \neq 1$.

Remark 3. Let $F$ be the complex line bundle over $V_{0} \cup V_{1}$ mentioned in Remark 2. In Example 1, we can easily verify that $F=[C] \mid V_{0} \cup V_{1}$. But this is not the case in general. We will show that, in Example 2, $[C]$ and $F$ do not coincide on any small neighborhood of $C$. To show this, let $C \subset S$ be as in Example 2 and suppose $[C]=F$ on some neighborhood of $C$. Then we have holomorphic functions $w_{i}$ on $V_{i}(i=0,1)$ such that $w_{1}=w_{0}$ on $V^{+}$and $w_{1}=\alpha w_{0}$ on $V^{-}$. Define a holomorphic 1 -form on $V_{0} \cup V_{1}-C$ by $\omega=d w_{i} / w_{i}^{\prime}$ on $V_{i}-U_{i}$ $(i=0,1)$. As shown in the Appendix, $\omega$ can be extended to a holomorphic 
1-form $\widetilde{\omega}$ on all of $S-C$. The restriction $\widetilde{\omega} \mid \pi^{-1}\left(q_{j}\right)$ has a pole with residue 1 at $C \cap \pi^{-1}\left(q_{j}\right)$ and holomorphic elsewhere. This contradiction shows the assertion.

\section{Appendix}

Theorem 3. Let $X$ be a 1-convex complex manifold of dimension 2, $K a$ compact set in $X$, and $\omega$ a closed holomorphic 1-form on $X-K$. Then $\omega$ can be extended to a 1-form $\widetilde{\omega}$ holomorphic on all of $X$.

First we show the following

Lemma 5. Let $E$ be a holomorphic vector bundle over $X$. Let $K$ be a compact set in $X$ and $s$ a holomorphic section of $E$ over $X-K$. Then $s$ can be extended to a meromorphic section $\tilde{s}$ over all of $X$. The set of poles of $\tilde{s}$ is a compact analytic set of dimension 1 in $X$.

Proof. Let $A$ be the maximal nowhere discrete compact analytic set in $X$. Let $A=\bigcup_{i=1}^{n} A_{i}$ be the decomposition of $A$ into irreducible components. By blowing up, we assume that the singularities of $A$ are normal crossings and that the components $A_{i}$ are non-singular. We can choose positive integers $p_{i}$ $(i=1, \cdots, n)$ so that the restriction of the line bundle $[D]=\sum_{i=1}^{n} p_{i}\left[A_{i}\right]$ to $A$ is negative. Hence, for sufficiently large $m$, we have $H^{1}\left(X, \mathcal{O}\left(K \otimes E^{-1} \otimes[D]^{-m}\right)\right)$ $=0$, by Ohsawa [6].

Now we consider the exact sequence

$$
H^{0}\left(X, \mathcal{O}\left(E \otimes[D]^{m}\right)\right) \stackrel{\mu}{\rightarrow} H_{\circ}^{0}\left(X, \mathcal{O}(E \otimes[D])^{m}\right) \rightarrow H_{*}^{1}\left(X, \mathcal{O}\left(E \otimes[D]^{m}\right)\right)
$$

where the subscript $\infty$ indicates the cohomology at infinity and $*$ indicates the cohomology with compact support. We have $H_{*}^{1}\left(X, \mathcal{O}\left(E \otimes[D]^{m}\right)\right)=0$, since it is dual to $H^{1}\left(X, O\left(K \otimes E^{-1} \otimes[D]^{-m}\right)\right)$ by Serre duality. Hence the mapping $\mu$ is surjective.

This shows that every holomorphic section $s$ of $E$ over $X-K$ can be extended to a meromorphic section $\tilde{s}$ over $X$, whose set of poles is contained in $A$.

q.e.d.

Proof of the theorem. By the lemma, $\omega$ can be extended to a meromorphic 1 form $\widetilde{\omega}$ on $X$. Let $A^{\prime}$ denote the set of poles of $\widetilde{\omega}$. We will show that $A^{\prime}$ is empty. 
Suppose that $A^{\prime}$ is non-empty. Let $A^{\prime}=\bigcup_{i=1}^{k} A_{i}$ be the decomposition of $A^{\circ}$ into irreducible components. Let $\left(r_{i j}\right)=\left(A_{i} \bullet A_{j}\right)$ be the intersection matrix of $\bigcup_{i=1}^{k} A_{\dot{i}}$. Since $A^{\prime}$ is an exceptional set, the matrix $\left(r_{i j}\right)$ is negative definite, by a theorem of Grauert [2].

Let $\alpha_{\dot{\xi}}$ denote the 1-cycle which goes around $A_{\dot{\xi}}$ in the positive sense $(i=1, \cdots, k)$. When regarded as elements in $H_{1}(X-A, \mathbb{Z})$, the 1 -cycles $\alpha_{i}$ are all torsion elements, since the relations

$$
\sum_{i=1}^{k} r_{i j} \alpha_{j}=0, \quad i=1, \cdots, k,
$$

hold by Mumford [3]. Hence we have

$$
\int_{\alpha_{i}} \tilde{\omega}=0, \quad i=1, \cdots, k \text {. }
$$

We choose a finite number of simply connected open sets $W_{\lambda}$ in $X$ such that $A^{\prime} \subset \cup W_{\lambda}$. By (4) there are meromorphic functions $f_{\lambda}$ on $W_{\lambda}$ such that $d f_{\lambda}=\widetilde{\omega}$. On $W_{\lambda} \cap W_{\mu}$, we have $f_{\lambda}-f_{\mu}=c_{\lambda \mu}$ (constant). Let $m_{i}(>0)$ be the order of poles of $f_{\lambda}$ on $A_{i}$, and define the divisor $D=\sum_{i=1}^{k} m_{i} A_{\dot{i}}$. Let $[D]$ be the line bundle associated to $D$ and let $s \in \Gamma(X, \mathcal{O}[D]))$ be the canonical section: $(s)=D$. The sections $s_{\lambda}=f_{\lambda} s \in \Gamma\left(W_{\lambda}-A^{\prime}, \mathcal{O}([D])\right)$ are extended to holomorphic sections $\left.\hat{s}_{\lambda} \in \Gamma\left(W_{\lambda}, \mathcal{O}[D]\right)\right)$. Since $\hat{s}_{\lambda}-\hat{s}_{\mu}=c_{\lambda \mu} S$ on $W_{\lambda} \cap W_{\mu}$, we can define a holomorphic section $\left.\hat{s} \in \Gamma\left(A^{\prime}, \mathcal{O}[D] \mid A^{\prime}\right)\right)$ by setting $\hat{s}=\hat{s}_{\lambda}$ on $A^{\prime} \cap W_{\lambda}$. The section $\hat{s}$ does not vanish on any component of $A^{\prime}$ and

$$
0 \leqq \operatorname{deg}\left(\hat{s} \mid A_{\dot{i}}\right)=D \cdot A_{\dot{i}}=\sum_{j=1}^{k} r_{i j} m_{j}, \quad(i=1, \cdots, k) .
$$

Hence $\sum_{i, j=1}^{k} r_{i j} m_{i} m_{j} \geqq 0$, which contradicts the fact that $\left(r_{i j}\right)$ is negative definite. Thus the theorem is proved.

\section{References}

[1] Enoki, I., Surfaces of class VII ${ }_{0}$ with curves. Tôhoku Math. J., 33 (1981), 453-492.

[2] Grauert, H., Über Modifikationen und exzeptionelle analytische Mengen, Math. Ann., 146 (1962), 331-368.

[ 3 ] Mumford, D., The topology of normal singularities of algebraic surfaces and a criterion for simplicity, Publ. Math. I.H.E.S., 9 (1961), 5-22.

[4 ] Narasimhan, R., On the homology groups of Stin spaces, Invent. Math., 2 (1967), 377385.

[ 5 ] Neeman, A., Ueda Theory: Theorems and problems, AMS Memoires, 415 (1989). 
[6] Ohsawa, T., Vanishing theorems on complete Kähler manifolds, Publ. RIMS, Kyoto Univ., 20 (1984), 21-38.

[ 7 ] Siu, Y.T., Every Stein subvariety admits a Stein neighborhood, Invent. Math., 38 (1976), $89-100$.

[ 8 ] Ueda, T., On the neighborhood of a compact complex curve with topologically trivial normal bundle, J. Math. Kyoro Univ., 22 (1983), 583-607. 
